INPLASY

PROTOCOL

To cite: Gao et al. Effects of pulmonary rehabilitation on exercise tolerance and quality of life in interstitial lung disease: a protocol for systematic review and metaanalysis. Inplasy protocol 202210064. doi: 10.37766/inplasy2022.1.0064

Received: 13 January 2022

Published: 13 January 2022

Corresponding author: Xiaoyan Gong

gongxiaoyan0817@163.com

Author Affiliation: Changchun University of Chinese Medicine.

Support: Jilin Science fund.

Review Stage at time of this submission: Preliminary searches.

Conflicts of interest:

None declared.

\section{Effects of pulmonary rehabilitation on exercise tolerance and quality of life in interstitial lung disease: a protocol for systematic review and meta-analysis}

\author{
Gao, Y1; Wang, S2; Yin, K3; Yuan, Y4; Gong, XY5.
}

Review question / Objective: Observed the effect of pulmonary rehabilitation or combining with another intervention on exercise tolerance and quality of life in interstitial lung disease compared with no pulmonary rehabilitation or another intervention, we aim to proved the efficacy of pulmonary rehabilitation to guide clinical practice. The type of studies are randomized controlled trials (RCTs). All eligible trials will be included regardless of language and publication type.

Condition being studied: The educational background, age and professional structure of the project team are reasonable. The members are young and middle-aged talents with rich clinical and scientific research experience. The personnel composition can meet the requirements of completing the project.

INPLASY registration number: This protocol was registered with the International Platform of Registered Systematic Review and Meta-Analysis Protocols (INPLASY) on 13 January 2022 and was last updated on 13 January 2022 (registration number INPLASY202210064).

\section{INTRODUCTION}

Review question / Objective: Observed the effect of pulmonary rehabilitation or combining with another intervention on exercise tolerance and quality of life in interstitial lung disease compared with no pulmonary rehabilitation or another intervention, we aim to proved the efficacy of pulmonary rehabilitation to guide clinical practice. The type of studies are randomized controlled trials (RCTs). All eligible trials will be included regardless of language and publication type.

Condition being studied: The educational background, age and professional structure of the project team are 
reasonable. The members are young and middle-aged talents with rich clinical and scientific research experience. The personnel composition can meet the requirements of completing the project.

\section{METHODS}

Participant or population: Participants must have a diagnosis of ILD and are in stable condition with no infection or exacerbation for at least the prior 4 months(most commonly will be idiopathic pulmonary fibrosis and without limitations related to gender, age, race, study area, and education status).

Intervention: In the intervention group patients received pulmonary rehabilitation or combined with another intervention.

Comparator: In the control group, patients received no treatment or another intervention.

Study designs to be included: randomized controlled trials (RCTs)

Eligibility criteria: Inclusion criteria were: (1)participants who had ILD (including pulmonary fibrosis); (2)pulmonary rehabilitation as the intervention; (3)randomized controlled trials (RCT); (4)exercise capacity, health-related quality of life.Exclusion criteria were: (1)participants have a diagnosis of ILD with other chronic respiratory diseases; (2)studies comparing the same modality of exercise with different doses of time, frequency, or duration;(3)conference abstracts without a full published article; (4)duplicated data or data that cannot be extracted.

Information sources: Two reviewers (YG and SW) independently searched the China National Knowledge Infrastructure(CNKI), the Chinese Biomedical Literature Database (CBM), Wanfang Data (WAN FANG), the Chinese Scientific Journal Database, the Cochrane Central Register of Controlled Trials, the Web of Science,PubMed and Embase data-bases from inception to December 8, 2021.Any disagreement will be resolved by discussion until consensus is reached or by consulting a third researcher(KY).We will also contact the original authors of papers via email or telephone if possible.The search items include diseases, intervention method and study type:("interstitial lung disease" or "interstitial pneumonia" or"pulmonary fibrosis"or"lung fibrosis" or"idiopathic pulmonary fibrosis") and ("pulmonary rehabilitation" or"respiratory rehabilitation" or"rehabilitation"or"respiratory muscle training"or"physical therapy"or"exercise training"or"exercise" or"endurance training"or"resistance training"or"flexibility training")and ("randomized controlled trial" or "randomized"or"trial").The reference lists of relevant research articles were reviewed for any additional article searches.

Main outcome(s): The primary outcomes were classified according to 2 main areas:exercise capacity measured using the 6-minute walk distance (6MWD); health-related quality of life(HRQL), measured using a quality of life scale.

Quality assessment / Risk of bias analysis: Two review authors (YG and SW) will assess the internal validity of included studies by using the Cochrane Handbook for Systematic Reviews of Interventions. The following domains will be assessed:random sequence generation and allocation concealment (selection bias), incomplete outcome data (attrition bias), outcome assessment(detection bias), selection of the reported result (reporting bias) and other bias. The intervention under evaluation in this metaanalysis is a non-drug-based therapy, making implementation of a placebocontrolled blinded study difficult.Thus, the term"blind method for study subjects"was removed.Results from these questions will be graphed and assessed using Review Manager 5.4.Each items will be evaluated in three categories: low risk of bias, unclear bias and high risk of bias. Any discrepancies will be resolved by reviewed the original article and discussed with the third author(KY) to reach a consensus. 
Strategy of data synthesis: Data will be subjected to statistical analysis using RevMan5.4 software.For continuous variables, we will present mean differences with standard deviations, or median and interquartile range.For dichotomous variables, we will report in the overall mean proportion (\%) of the population.The $95 \%$ $\mathrm{Cl}$ will be presented for both dichotomous and continuous outcomes.Statistical heterogeneity will be assessed with the 12 statistic.We will interpret it using the following criteria: 12 values of $25 \%$ is considered low levels of heterogeneity, $50 \%$ indicated moderate levels, and $75 \%$ indicated high levels.Since low or moderate heterogeneity suggests little variability among these studies, the data will be analyzed in a fixed-effects model.When significant heterogeneity occurs among the studies (P50\%), a random-effect model will be performed to analyze the data.

Subgroup analysis: We plan to carry out the following subgroup analyses to explore possible sources of heterogeneity: type of interstitial lung disease, severity of ILD, course of disease, type of exercise and treatment duration.If the data is insufficient, qualitative synthesis will be conducted instead of quantitative synthesis.

Sensitivity analysis: When possible,we will perform sensitivity analysis by eliminating low quality trials to explore the effects of the trial's bias risk on primary outcomes.

Country(ies) involved: China.

Keywords: Interstitial lung disease; Pulmonary rehabilitation; exercise tolerance; quality of life; systematic review; meta-analysis; protocol.

Contributions of each author:

Author 1 - Ying Gao - YG conceived the study, provided general guidance to the drafting of the protocol, reviewed and revised the manuscript.

Email: gy02701010@163.com

Author 2 - Shuang Wang - SW drafted the protocol and manuscript.

Email: wangshuang220112@163.com
Author 3 - Kai Yin - KY drafted the protocol and manuscript.

Email: yinkai1113@163.com

Author 4 - Yue Yuan - YY designed the search strategy.

Email: yuanyue9210@163.com 